\title{
Social media, importância no turismo de afinidades
}

\author{
Social media, importance in affinity tourism
}

\author{
Maria Celeste Cantante \\ Universidade Aberta, Lisboa, Portugal \\ celestecantante@gmail.com
}

\begin{abstract}
Resumo
O propósito deste estudo prende-se com as social media e a sua importância no turismo. A necessidade de aglutinar e partilhar informações em redes seguras, durante a Segunda Guerra Mundial, desenvolveu um processo de redes de comunicação que culminou com a World Wide Web, nos anos 80 . As vivências multiculturais dos soldados e dos refugiados da guerra e a melhoria das condições de vida desencadearam o desejo de viajar, ensejo que as redes sociais ajudam a concretizar influenciando o turismo. O objetivo deste trabalho é evidenciar em que medida as social media contribuíram para o crescimento do turismo, que beneficia da partilha de experiências de lazer, cria proximidades e contraria a padronização, marcando o desenvolvimento de um turismo de afinidades que aproxima e satisfaz.
\end{abstract}

Palavras-chave: Social media; proximidade; afetividade; turismo; partilha.

\begin{abstract}
This study focuses on social media and its importance to tourism. The need to gather and share information via secure networks during World War II developed a process of communication networks that culminated in the World Wide Web in the 1980s. The multicultural experiences of soldiers and war refugees and the improvement of living conditions triggered the desire to travel, an opportunity that social networks help to achieve by influencing tourism. In this paper we aim to highlight the extent to which social media has contributed to the growth of tourism, which benefits from the sharing of leisure experiences, creates proximities and goes against standardization, marking the development of a tourism of affinities that brings people together.
\end{abstract}

Keywords: Social media; proximity; affectivity; tourism; sharing.

\section{Introdução}

A origem da sociedade de informação remonta à Segunda Guerra Mundial, considerando-se a Guerra Fria a responsável pela transformação do mundo, com o surgimento da Internet. A partir dos anos de 1980, as World Wide Web desencadearam uma necessidade de informação e comunicação jamais verificada, transformando o modus vivendus do cidadão comum, em particular e da sociedade, em geral.

Com a partilha de experiências vividas durante a Guerra, nomeadamente espaços e culturas desconhecidas, surgiu a necessidade de viajar, de conhecer novas gentes e lugares, de fazer turismo. A Internet, mas, sobretudo as redes sociais, em resultado da partilha de experiências online, contribuem para a divulgação de espaços e de gentes, bem como da qualidade dos serviços prestados. Deste modo, influenciam escolhas, constituindo uma 
enorme mais-valia para o turista, uma vez que lhe desencadeia uma apetência maior para vivenciar experiências similares.

A par de facilitadores do aumento do turismo, as social media aproximam os indivíduos, desenvolvendo um movimento que se transforma numa nova forma de fazer turismo, criando um "turismo de afinidades" decorrente dos benefícios das redes sociais, particularmente refletido no aumento do grau de confiança da informação obtida.

Uma proximidade comunicacional virtual parece ser, cada vez mais, um instrumento relacional imprescindível na vida quotidiana, verificando-se uma alteração de paradigma que lança um desafio maior e põe à prova as tradicionais formas de "fazer turismo". Refletir sobre as social media e a sua importância no "turismo de afinidades" constitui uma necessidade que as práticas do cidadão comum reclamam.

\section{Breve percurso transformativo do conceito de turismo}

A Segunda Guerra Mundial desencadeou um processo na área da comunicação que transformou a sociedade e o seu modus vivendus, sendo considerada o berço da Sociedade de Informação. Porém, foi com a Guerra Fria que a Internet surgiu e, sobretudo a partir dos anos oitenta, o mundo mudou de forma irreversível.

Os fenómenos de beligerância agregam necessidades de aglutinar e fazer circular informações com vista à defesa, à inversão das posições dos intervenientes dos quadros de guerra, à conquista de territórios. Durante a Segunda Guerra Mundial, a necessidade de comunicação sigilosa em redes seguras proporcionou o desenvolvimento de instrumentos, que a tornaram possível e com menores riscos de descoberta de estratégias militares que poderiam fazer perigar o sucesso das forças aliadas, em beligerância. O período da Guerra Fria, marcada pela espionagem e pelo periclitante equilíbrio de poderes, incrementou $\mathrm{o}$ interesse pelas redes de informação e comunicação, o que permitiu o surgimento de sistemas, progressivamente mais sofisticados, que desencadearam pesquisas académicas e privadas, delas sobressaindo a criação de redes globais de computadores, tendo Tim BernersLee fundado a World Wide Web, no final dos anos oitenta. Na atualidade, "a Internet é a maior rede de comunicação desenvolvida pelo homem na era moderna." (Canarim, 2012).

Constata-se que os soldados da Segunda Grande Guerra trouxeram consigo experiências pessoais em locais desconhecidos, uma vez que, "military service forced many people who had never before traveled to do so, and they were eager to share their positive experiences with family and friends when they returned home" (Gade \& Ankathi, 2016: 52). Estes factos conduziram ao desejo do seu conhecimento. O pós-guerra, favorecido pela prosperidade dos anos 1950 e 1960, pelo cartão de crédito e pelo jet travel, determinou o desenvolvimento do turismo de massas, em oposição ao turismo de elites dos princípios do século XX. A introdução do subsídio de férias aos trabalhadores por conta de outrem facilitou o incremento do turismo. O surgimento e o desenvolvimento da WWW, nas décadas de $1980 \mathrm{e}$ 1990, são fatores associados à transformação do conceito de turismo. Na atualidade, "more than 500 million wage-earners are entitled to paid holidays." (Gade \& Ankathi, 2016: 81). A acrescentar aos fatores que contribuíram para a mudança do conceito de turismo, deve ainda referir-se "the impact of the modern transport technology, especially in the aviation field [that] has brought a completely new meaning to travel tourism." (Gade \& Ankathi, 2016: 84), 
o que tem vindo a sedimentar uma conceção de turismo acessível às diferentes camadas sociais.

A palavra turismo passou a fazer parte dos hábitos das várias classes sociais, assumindo, nos últimos tempos, uma importância desconhecida até aqui. Um pouco por todo o lado, nomeadamente no mundo ocidental, tem-se vindo a enraizar o hábito de praticar turismo, do cidadão se deslocar e procurar o prazer em outros lugares, fugir às rotinas, criar alternativas temporárias de maior satisfação pessoal.

Tourism is concerned with pleasure, holiday, travel and going or arriving somewhere. (...) Tourism is concerned with consuming goods and services. (...) Tourism, as a significant social phenomenon, involves a temporary break with normal routine to engage with experiences that contrast with everyday life. (...) Tourism has also become a status symbol in modern society and thought to be necessary to one's health. (Gade \& Ankathi, 2016: 33-36)

Uma mudança significativa dos padrões de vida, nomeadamente os meios de transporte, cada vez mais céleres e sofisticados, o conceito de estatuto social, a sociedade de consumo, a celeridade da vida quotidiana, obrigam a uma adaptação, à mudança de paradigmas, à mudança dos tempos e dos hábitos do mundo global em geral, e da atividade turística em particular.

Todavia, é nos media que reside a causa de uma significativa mudança de paradigma. A internet, as networks, as social networks, as social media estão presentes nas mais diversas áreas tornando-se imprescindível a sua utilização. Através dos media o cidadão comum tem acesso aos mais variados "mundos" de hábitos e culturas, despoletando o desejo do seu conhecimento.

\section{Social media - Uma ferramenta que marca o turismo de afinidades}

A visibilidade da importância das social networks repercute-se na indústria turística. A uma mudança de paradigma na área do turismo, estão associadas as agências de turismo que desempenham um importante papel na satisfação das necessidades do turista que a elas recorre com frequência, muitas vezes sugestionado pelos utilizadores das social media, onde os amantes do lazer ou da aventura exprimem opiniões e relatam experiências. Com efeito, verifica-se que "social media has made a huge impact on the tourism industry. Consumers engage with social networking sites to research trips, make informed decisions about their travels and share their personal experiences of a particular hotel, restaurant or airline" (Mangan, 2015: s.p.). De facto, o enorme impacto que as social media têm na vida social, numa nova época marcada por uma socialização online, repercute-se também no turismo. "The use of Internet and other information communication technologies leads to a new era of tourism economy. Social media, as one of most powerful online networking tools, has been integrated into a part of social and economic life in the real world" (Zeng, 2012: 1).

As social media são, portanto, e cada vez mais, uma ferramenta imprescindível, influenciando os mais variados aspetos da vida social e o turismo em particular, que deles tira vantagens.

As social media desempenham um papel relevante numa relação de socialização e comunicação entre os seus utilizadores, criando laços de proximidade que os tornam 
indissociáveis da criação de opinião, em parte sustentada pela partilha de experiências. A celeridade da vida quotidiana exige uma resposta a que os media em geral, e as social media, em particular, procuram corresponder, impondo-se como força catalisadora de proximidade, advindo desta realidade benefícios de ordem relacional.

As social media encurtam distâncias propiciando um processo de socialização virtual, online, através da comunicação e da imagem. A proximidade, embora virtual, dos intervenientes no processo de comunicação pela Internet contribui para o aumento de confiança, constituindo-se um facilitador relacional de onde resultam benefícios de vária ordem, dos quais destacamos os funcionais e os sociais, que atribuem às social media um papel facilitador do consumo turístico. As conversas através dos meios online desencadeiam o desejo de fazer amigos, de partilhar informações, de conhecer espaços, de vivenciar aventuras, de correr riscos: "Os sítios, os lugares, os territórios adquirem novas dimensões, simbólicas e imagéticas, por um lado, mas também de proximidade, proteção e afetivas, por outro alado. Ao turismo de massas justapõe-se um turismo de nicho, [...] de elevado nível de personalização contrapondo o "velho" turismo de massas" (Cravidão \& Santos, 2013: 10).

O turismo de "nicho", facilitado pelas social media, transforma e direciona a experiência turística numa oportunidade de enriquecimento pessoal, num trânsito recíproco educativo, num fator de socialização privilegiado que contempla a aproximação e, por consequência, as afinidades e os afetos, acrescentando-Ihe criatividade e marcando no cidadão uma etapa na sua formação, na forma como, facilmente, aprende a partilhar os seus universos. "O turismo, na sua versão criativa, é hoje apresentado como um fator emancipatório e marca de cidadania experimental. [... ] na situação de quem participa diretamente no universo íntimo do "outro", do seu trabalho ou das suas experiências quotidianas." (Cravidão \& Santos, 2013: 277) contrapondo a um conceito de massas, vazio de proximidades, uma conceção de aproximação e de intimidade resultante da comunicação fácil e socialilizante que se estabelece através das social media.

A comunicação instantânea, que as social media permitem, apresenta vantagens do domínio do aumento da visibilidade de um espaço de lazer, a emoção de uma experiência partilhada, o deslumbramento de uma paisagem, quebrando constrangimentos, divulgando e aliciando para locais turísticos ao alcance do cidadão comum que neles se revê e procura as suas próprias experiências e emoções. A capacidade de divulgar histórias verosímeis e aliciantes de "aventuras" de lazer das social media, contribui para o aumento da visibilidade de locais e serviços turísticos, aumentando a sua procura, contribuindo para "an ongoing trend where hospitality has a lot to gain." (Benea, 2014: 44).

Por conseguinte, constata-se que as social media são facilitadoras de uma "ongoing connection and relationship with the guests [that] will eventually turn them into embassadors and a volunteer marketing army" (idem ibidem), o que, por um lado, contribui para o aumento da procura de locais de lazer mais hospitaleiros e, por consequência, para a ascensão do turismo, por outro, poderia transformar as social media em "agências de turismo" gratuitas, sem controlo e sem responsabilização, correndo-se o risco de desprestigiar o turismo e a hotelaria, o que poderia lesar os seus usufrutuários.

No entanto, apesar de alguns riscos que as social media representam, "the last decade has seen a significant increase in the use of both social media and the general development of new technology. [...] The tourism and hospitality industry has seen an impressing development since the beginning" (Benea, 2014: 7), sendo as social media utilizadas 
enquanto instrumento de busca: "social media plays a significant role in many aspects of tourism, especially in information search and decision-making behaviours, tourism promotion and in focusing on best practices for interacting with consumers. Leveraging off social media to market tourism products has proven to be an excellent strategy" (Zeng, 2014: 1).

O mercado do turismo tem vindo, de facto, a beneficiar das redes sociais utilizadas como estratégia eficaz no aumento da procura de locais e serviços.

Juliet Carnoy (2016), em 5 ways social media has transformed tourism marketing, refere o modo como este instrumento online tem vindo a alterar os mecanismos de promoção do turismo, nos tempos mais recentes. Em primeiro lugar temos as social media como instrumento de busca em resultado da divulgação de locais e de serviços prestada pelo cidadão comum, que favorece o marketing low cost e, por consequência, o consumidor. Com efeito, os potenciais turistas "can easily find other travelers' photos, check-ins, ratings" (Carnoy, 2016). utilizando este meio online, o que facilita, fomenta e sugestiona as suas opções, uma vez que dispõe, previamente, de opiniões que favorecem as decisões a tomar, constituindo, portanto, uma fonte de inspiração.

A autora Carnoy (2016) considera também que com esta ferramenta se assiste a um incremento da partilha de experiências de turismo online, o que pode influenciar escolhas e contribuir para a melhoria dos locais de oferta que, sabendo-se expostos, procuram transmitir imagens de qualidade e eficiência de serviços, saldando-se este modelo de partilha numa mais-valia para o consumidor. No ponto de vista dos serviços hoteleiros, esta nova e económica fonte de divulgação pode ser encarada como um benefício e um controlo de satisfação do cliente.

As social media podem ainda influir no grau de satisfação do utilizador no que respeita a hotéis, resorts e outros espaços ao serviço do viajante turístico, na medida em que os bons serviços disseminados promovem os espaços a preferir, do mesmo modo que os comentários menos abonatórios prestam um bom serviço na melhoria das condições de oferta. A interação que se estabelece, a partir destas experiências de partilha, "can help [... ] create an exceptional experience that is sure to delight [... ] guests" (Carnoy, 2016.).

Uma outra questão a evidenciar diz respeito ao aumento da percentagem de reserva direta, consequência da influência das social media, o que resulta num impacto nas agências de turismo que se vêm na contingência de melhorar os seus serviços e de utilizar, com maior frequência modelos digitais para reserva, adaptando-se a novas tecnologias. Carnoy (2016) salienta que os "loyalty programs have become a core piece of the travel business model, and social media has had a massive impact on how hotel loyalty programs are constructed" (idem ibidem), concluindo que "guests feel entitled to compensation for the positive wordof-mouth marketing" (idem ibidem), o que coloca, também as social media em lugar de destaque na apreciação e na divulgação do grau de satisfação do cliente.

Importa, portanto, realçar a influência das social media na alteração e melhoria das políticas de mercado da indústria hoteleira e de oferta de espaços e serviços de lazer, bem como influem num maior grau de confiança nas escolhas do turista. Por conseguinte, deve destacar-se que "the advantages of social media should not only be considered from the corporation point of view, but also from the consumer point of view. Thanks to the internet consumers can reach out more options and social media is na invaluable communication médium among the consumers" (Katsoni, 2014: 246). 
O Instagram, o Pintarest, o Facebook, o Twitter, o Linkedin, entre outros, contribuem para que se criem laços e relacionamentos, aproximam pessoas, aprofundam relações pela interação que facilitam e o negócio do turismo tem vindo a beneficiar destas ferramentas que contribuem para uma mudança de paradigma no domínio das relações interpessoais, em geral, e do turismo, em particular.

O efeito das redes sociais sobre o ato comunicativo ao serviço do cidadão comum, representa um enorme potencial de descoberta de destinos, fomenta e inspira opções suportadas por graus de confiança significativos, decorrentes da inter-relação próxima e frequente. O desempenho das social media no processo de busca do lazer, apresenta-se cada vez mais influente na dinâmica de uma nova forma de "fazer turismo", motivando, cada vez mais, para a sua utilização, uma vez que passou a constituir parte integrante do quotidiano, apresentando-se eficaz e um modelo de confiança em muitos países com tradição de destino turístico, como é o caso de Portugal.

Pelas condições geográficas, de clima e de segurança que apresenta, Portugal constitui um recanto cada vez mais procurado por muitos indivíduos e famílias que, numa fuga ao quotidiano insustentável da atualidade, procuram a reposição da tranquilidade e do tempo de lazer, representando o turismo, cada vez mais, uma mais-valia para o país, que se vê assolado por um boom de procura jamais vivenciado, razão pela qual "Portugal valoriza cada vez mais o turismo como factor decisivo para a riqueza nacional, fazendo sentido neste momento pensar a melhor maneira de rentabilizar os recursos disponíveis de forma a amplificar ainda mais o destino turístico Portugal nos mercados estrangeiros" (Anjos, 2014: 24).

A globalização, cuja dinâmica de interação aproxima pessoas e difunde o mundo à velocidade de um click. "A globalização [...] definida como o processo de interacção e integração entre as pessoas, as empresas e os diversos governos" (Duarte, Fontes, Franco \& Almeida, 2009) não sobreviveria sem as redes sociais que satisfazem muitas das necessidades da sociedade global, que descobrem e aproximam os locais mais recônditos do planeta, despertando para o desejo de experiências in loco, levando as agências turísticas à sua divulgação e as empresas turísticas a explorar as suas potencialidades.

Parte integrante do processo de globalização, as social media desenvolvem um movimento sem retorno estimulado pela aproximação que elimina barreiras de pessoas, de serviços, de espaços, de produtos, aumentando a eficácia da comunicação, num processo de transformação que contribui para "para a melhoria dos níveis de conforto das populações." (Duarte et al., 2009), o que tem "contribuído para a afirmação do turismo internacional" (idem ibidem). O turismo em Portugal, procurando corresponder aos novos tempos, tenta dar-se visibilidade numa era global que tudo vê e tudo busca. Atento a uma procura sem precedentes, o turismo em Portugal projeta-se em leis facilitadoras do seu crescimento, no desenvolvimento dos meios de transporte, no aumento dos espaços de lazer. O fenómeno "globalização funciona como um forte estímulo à actividade turística" (idem ibidem) e Portugal não é alheio a este fenómeno. Associada às social media, esta atividade é facilitadora da divulgação da História, da cultura e das tradições locais disseminando-se, deste modo, os hábitos e os costumes dos povos e Portugal não é exceção.

Paralelamente, interessa destacar que as redes sociais, a par do papel importantíssimo que desempenham no desenvolvimento do turismo, podem ser incentivadoras dos chamados "guetos turísticos", nos quais "as populações locais são relegadas para segundo 
plano, sendo privilegiada a estada dos turistas, à custa do uso e abuso dos recursos locais. Assim, muitas das vezes o que se pretendia alcançar pelo impulsionamento da actividade turística acaba por nunca se concretizar. Pelo contrário, é agravado ainda mais o fosso que separa as duas realidades" (idem ibidem).

Os chamados "guetos turísticos" oferecem "vivências" muitas vezes "[... ] remetidas para uma certa «folclorização» da cultura local [... ] despojadas de qualquer essência" (idem ibidem). Uma prática desta natureza empobrece a relação afetiva que se poderia estabelecer com o local escolhido para férias ou pequenas escapadas, o que não beneficia ambas as partes, podendo mesmo gerar "relações de conflito e de desagrado entre os visitantes e os visitados, muitas das vezes com consequências sérias. Os turistas por seu turno não geram afinidades com os destinos e não se fidelizam." (idem ibidem). Em Portugal, assistimos, durante anos, a uma prática que relegou a imagem do turismo nacional para uma conceção pouco profissional, situação que tem vindo a alterar-se, graças, em parte, ao papel que as social media desempenham na partilha de opiniões e na disseminação de espaços e culturas propiciando um enorme afluxo de visitantes que, exigindo maior qualidade, obrigam a melhores ofertas.

Uma outra questão que se coloca prende-se com a implementação de marcas internacionais em território português. Se por um lado, asseguram uma qualidade testada, por outro, podem desprestigiar a oferta nacional, estimulando, de modo similar, pela concorrência que geram, melhoramentos da oferta nacional, podendo as social media assumir-se como fiel de uma balança de interesses que o mundo global coloca em igualdade de oportunidades.

Afigura-se, no entanto, claro que as vantagens se sobrepõem aos constrangimentos, uma vez que a partilha de opiniões e de experiências que as social media disseminam favorece uma forma de turismo de proximidade que privilegia as relações afetivas, assistindo-se, em Portugal, ao crescimento de um "turismo de afinidades", graças a hábitos enraizados na forma de estar dos portugueses sedimentados na partilha e nos afetos espalhados pelas redes sociais que beneficiam uma procura fortalecida pelos pareceres divulgados, que têm contribuído para divulgar Portugal como local a procurar, atribuindo-lhe a segurança e os afetos que o tornam atrativo.

O grupo etário mais intimamente ligado às redes sociais é a camada juvenil que, mais sensível e de emoções fortes, difunde e partilha impressivamente as suas experiências diárias nas redes sociais, associando-se ao turismo de afinidades o turismo juvenil que, pelas suas caraterísticas de turismo social e associativo (intercâmbios educativos e os Albergues da Juventude), contribui para a criação de laços afetivos e relacionamentos mais consistentes e duradouros e, consequentemente, para a divulgação de locais, gentes e culturas, nele se evidenciando "como pontos fortes a qualidade de animação e capacidade de convivência de união de grupos com as mesmas afinidades" (Giaretta, 2003: 16).

A alteração do mapa turístico tradicional em resultado das condições de insegurança internacionais tem, de modo similar, favorecido o turismo em Portugal.

A segurança oferecida, bem como as características locais, divulgadas nas redes sociais, constituem fatores importantes ponderados nas escolhas dos visitantes, sendo este fenómeno de divulgação online aproveitado para um planeamento que privilegia uma oferta de segurança, de inspiração local vocacionada para o ato comunicativo e a socialização. A uma maior procura associa-se, igualmente, uma oferta de proximidade que se opõe à 
indiferença que distancia as relações sociais e a comunicação que caracteriza a sociedade atual, revertendo uma tendência de isolamento.

\section{Conclusão}

As social media representam uma sustentável mais-valia na divulgação de espaços de lazer seguros e credíveis que o mundo atual oferece, fustigado por atentados e conflitos bélicos que alteraram o mapa turístico tradicional. Este fenómeno informático contribui para a sustentabilidade de uma oferta turística que projeta os países além-fronteiras. A proximidade, que o comentário online sustenta, contribui para o sucesso do setor turístico, se aproveitado para um melhor planeamento. As social media, pelas suas características de proximidade e de visibilidade, garantem uma atratividade que o passa palavra promove, embora apresente alguns riscos, como a criação dos "guetos turísticos".

As social media podem contrariar uma tendência "folclorista", pela contribuição num planeamento sustentado que evidencie o que de melhor Portugal pode oferecer. Uma oferta suportada num profissionalismo decorrente da partilha de opiniões, um turismo de inspiração local que prioriza a segurança e os afetos, um turismo de afinidades que as social media divulgam, parece ser um caminho que o turismo em Portugal sabiamente vem a percorrer, valorizando, por oposição à padronização, uma relação de proximidade com o cliente.

\section{Referências}

Anjos, C. (2014). Análise de media e desempenho da comunicação do turismo de Portugal no estrangeiro. Os casos do Reino Unido, França e Espanha (Relatório de estágio de mestrado não publicado). Instituto Superior de Ciências Sociais e Políticas da Universidade de Lisboa, Lisboa.

Benea, J. A. (2014). Influences of social media on the tourism and hospitality industry (unpublished Master's thesis). MODUL University Vienna, Vienna.

Canarim, P. (2012). O nascimento da internet começou na 2. ${ }^{a}$ Guerra Mundial. Disponível em http://webinsider.com.br/2012/04/07/o-nascimento-da-internet-comecou-na-2a-guerramundial/.

Carnoy, J. (2016). 5 ways social media has transformed tourism marketing. Disponível: https://www.entrepreneur.com/article/286408

Cravidão, F. \& Santos, N. (2013). Turismo e cultura: Destinos e competitividade. Coimbra: Imprensa da Universidade de Coimbra.

Duarte, G., Fontes, A., Franco, R. \& Almeida, H. (2009). A globalização e o turismo. Publituris. Disponível em: https://www.publituris.pt/2009/11/11/a-globalizacao-e-o-turismo/

Gade, J. \& Ankathi, R. (2016). Tourism management philosophies, principles and practices. India: Zenon Academic Publications.

Giaretta, M. J. (2003). Turismo de juventude. Brasil: Editora Manole.

Katsoni, V. (Ed.) (2014). Cultural tourism in a digital era. Proceedings of the First International Conference IACuDiT. Greece: Springer.

Mangan, M. (2015). The impact of social media on the tourism industry. Hospitalitynet. Disponível em: https://www.hospitalitynet.org/news/4071855.html

Zeng, B. (2012). Social media in tourism. Disponível em: https://www.omicsonline.org/openaccess/Social-Media-in-Tourism-2167-0269.1000e125.php?aid=19014.

Zeng, B. (2014). Tourism management perspectives what do we know about social media in tourism? A review. Disponível em: https://researchers.cdu.edu.au/en/publications/what-do-we-knowabout-social-media-in-tourism-a-review. 
MARIA CELESTE CANTANTE é doutora em Literatura, Especialidade em Literatura Norte-Americana, investigadora FCT do Grupo de Investigação: CEMRI - Media e Mediações Culturais, Universidade Aberta, professora de Inglês de quadro de agrupamento. Tem apresentado várias comunicações a nível nacional e internacional: Universidade Aberta Porto e Lisboa - CEMRI; Universidade do Minho; Escola Superior de Educação do Instituto Politécnico de Viana do Castelo - Associação Ao Norte; Universidade Presbiteriana Mackenzie; Faculdad de Comunicación Universidad de Sevilla, tendo a maioria sido publicada. Workhops na Escola Superior de Educação do Instituto Politécnico de Viana do Castelo, a mestrandos. Supervisão de dissertação de Mestrado do mesmo curso em colaboração com a orientadora, a seu convite (a decorrer). Endereço institucional: Agrupamento de Escolas José Afonso de Alhos Vedros - Bairro das Morçoas, 2860 - Alhos Vedros, Portugal.

Submetido em 12 novembro 2017

Aceite em 3 abril 2018 